\title{
Automated Human Identification Scheme using Ear Biometrics Technology
}

\author{
V.K. Narendira Kumar ${ }^{1}$ and B. Srinivasan ${ }^{2}$ \\ ${ }^{1}$ Assistant Professor, Department of Information Technology, \\ ${ }^{2}$ Associate Professor, PG \& Research Department of Computer Science, \\ Gobi Arts \& Science College (Autonomous), Gobichettipalayam - 638 453, Erode District, Tamil Nadu, India. \\ ${ }^{1}$ kumarmcagobi@yahoo.co, ${ }^{2}$ srinivasan_gasc@yahoo.com
}

\begin{abstract}
Biometrics identification methods have proved to be very efficient, more natural and easy for users than traditional methods of human identification. Biometrics methods truly identify humans, not keys and cards they posses or passwords they should remember. Ear on the other hand, has a more uniform distribution of color, so almost all information is conserved when converting the original image into gray scales. We propose the ear as a biometric and investigate it with both 2D and 3D data. The ICP-based algorithm also demonstrates good scalability with size of dataset. These results are encouraging in that they suggest a strong potential for 3D ear shape as a biometric. Multi-biometric $2 \mathrm{D}$ and $3 \mathrm{D}$ ear recognition are also explored. The proposed automatic ear detection method will integrate with the current system, and the performance will be evaluated with the original one. The investigation of ear recognition under less controlled conditions will focus on the robustness and variability of ear biometrics. Multimodal biometrics using 3D ear images will be explored, and the performance will be compared to existing biometrics experimental results.
\end{abstract}

Index Terms - Ear, Biometrics, Recognition, Detection and Extraction.

\section{INTRODUCTION}

Biometrics is human identifications by measuring physical or behavior characteristics of a person to verify his identity. Public safety and national security magnify the need for biometric technique, which are amongst the most secure and accurate authentication tools. Ear images can be acquired in a manner similar to face images, and at least one previous study suggests they are comparable in recognition power. Additional work on ear biometrics may lead to increased recognition flexibility and power in such scenarios [3].

Many research studies have proposed the ear as a biometric. In fact, the ear may already be used informally as a biometric. For example, the United States Immigration and Naturalization Service (INS) have a form giving specifications for the photograph that indicates that the right ear should be visible. Researchers have suggested that the shape and appearance of the human ear is unique to each individual and relatively unchanging during the lifetime of an adult. No one can prove the uniqueness of the ear, but two studies mentioned in provide empirical supporting evidence. The medical report shows that the variation over time is most noticeable during the period from four months to eight years old and over 70 years old. The ear growth between four months to eight years old is approximately linear, and after that it is constant until around 70 when it increase again. The stretch rate due to gravity is not linear, but it mainly affects the lobe of the ear. Due to its stability and predictable changes, ear recognition is being investigated as potential biometric. Generally, ear images can be acquired in a manner similar to face images, and used in the same scenarios.

A biometric system is essentially a pattern recognition system which uses a specific physiological or behavioral characteristic of a person to determine the person's identification. Therefore, a biometric system can be solved using the methodologies from the pattern recognition research. Researcher considers the use of both 2D and 3D images of the ear, using data [1].

The work presented in this proposal is unique in several points. We report results for the largest ear biometrics study to date, in terms of number of persons. Ours is the only work to compare $2 \mathrm{D}$ and $3 \mathrm{D}$ ear recognition and compare multiple different algorithms for $3 \mathrm{D}$ ear recognition. We are applying an ICP-based approach to 3D ear recognition. Only one other work that we are aware of considers 3D ear biometrics, and here we compare the results of that approach to three other approaches for 3D ear biometrics. Because we use a large experimental dataset, we are also able to explore how the different algorithms scale with number of persons in the gallery.

The remaining sections are organized as follows: Brief outline of Ear Biometric is presented in section 2. Ear preprocessing methodology is mentioned in Section 3. The other phases of the implementations of the ear biometrics are briefly explained in section 4 . The testing and performance of ear biometrics is mentioned in Section 5. Experimental results are given in Section 6. Finally, Section 7 describes the concluding remarks.

\section{LITERATURE REVIEW}

As the mentioned before, many research studies have 
proposed the ear as a biometric. Researchers have suggested that the shape and appearance of the human ear is unique to each individual and relatively unchanging during the lifetime of an adult. There are several studies that attempt to solve the question of uniqueness and classification of ears. No one can really prove the uniqueness of the ear, but two studies mentioned in provide empirical supporting evidence. In 1906, Imhofer already found that only 4 characteristics were needed to distinguish a set of 500 ears. The most prominent work is done by Iannarelli. In his work, over 10,000 ears were examined and no indistinguishable ears were found. Iannarelli developed an anthropometric technique for ear identification.

Bhanu and Chen presented a 3D ear recognition method using a local surface shape descriptor. The local surface patches are defined by the feature point and its neighbors, and the patch descriptor consists of its centroid, 2D histogram and surface type. There are four majors' steps in the method: feature point extraction, local surface description, o_-line model building and recognition. Twenty range images from 10 individuals ( 2 images each) are used in the experiments and a $100 \%$ recognition rate is achieved for their dataset.

Researcher implemented their method from the description in. Slight differences were determined experimentally: (1) Due to the noisy nature of range data, the feature points are determined by the shape index type instead of the shape index value. (2) Considering the computation time required, comparison of the two local surfaces was done only when their Euclidean distance was less than 40 pixels. This assumption is valid in the dataset. Using two images each from the first 10 individuals in the dataset, researcher also found a $100 \%$ recognition rate. But when researcher increased the dataset to 202 individuals, the performance dropped to $33 \%$ (68 out of 202). The computation time required for this technique was also larger than that for PCA-based and edge-based techniques that researcher investigated.

\section{A. Ear Detection}

Given a still 2D or 3D image, ear detection is defined as the localization of the regions that contain a human ear regardless of its size, orientation and hair occlusion. Ear recognition is either ear identification or ear verification. Both of them assume that the ears have been already extracted from an image or at least have already been localized. So ear detection is the preliminary step in automatic ear recognition systems, and it is essential to recognize the ear correctly and efficiently [2].

Similarly, face detection is a challenging task, but has been widely explored. A detailed survey of face detection work can be found in [1]. The challenges associated with face detection are differences in variation among pose, the presence or absence of structural components, facial expression, occlusion, image orientation and image conditions [5]. Among these factors, some are characteristics that only belong to the face. But some factors, like pose, occlusion, image orientation and image conditions also appear in the ear detection problem.
Therefore, it will be interesting to apply some face detection algorithms to detect ear.

\section{B. Approaches}

Approaches considered include a PCA ("eigen-ear") approach with 2D intensity images, a PCA (Principal Component Analysis) approach with range images, Hausdorff matching of edges from the range images, and ICP based matching of the 3D data. Researcher also performed initial experiments with the own implementation of an ear shape matching algorithm due to Bhanu and Chen. But the performance drops dramatically when researcher increased the dataset size from 10 to 202 .

A PCA ("Eigen-ear") approach with 2D intensity images, achieving $63.8 \%$ rank-one recognition; a PCA approach with range images, achieving 55.3\%; and Hausdorff matching of edges from range images, achieving $67.5 \%$. Starting from the general ICP algorithm proposed by, researcher obtained an $84.3 \%$ rank-one recognition rate on $3 \mathrm{D}$ ear biometrics. The ICP-based approach not only achieves the best recognition performance of the various methods that researcher considered, it also shows good scalability with size of dataset. The promising experimental results of the ICPbased approach suggests the strong potential for 3D ear shape as a biometric, and also encouraged us to investigate the ICP algorithm both for performance and for computational time.

\section{ICP Algorithm}

Three algorithms have been explored on 2D and 3D ear images, and based on that, three kinds of multi-biometrics are considered: multi-modal, multi-algorithm and multiinstance. Various multi-biometric combinations all result in improvement over a single biometric. Multi-modal 2D PCA together with 3D ICP gives the highest performance. To combine 2D PCA-based and 3D ICP-based ear recognition, a new fusion rule using the interval distribution between rank one and rank two outperforms other simple combinations. The rank one recognition rate achieves $91.7 \%$ with 302 subjects in the gallery. In general, all the approaches perform much better when multiple images are used to represent one subject. In the dataset, 169 subjects had 2D and 3D images of the ear acquired on at least four different dates, which allowed us to perform multi-instance experiments. The highest rank one recognition rate was $97 \%$ with the ICP approach used to match a two-image-per-person probe against a twoimage-per-person gallery. In addition, researcher found that different fusion rules perform differently on different combinations. The min rule works well when combining the multiple presentations of one subject, while the sum rule works well when combining multiple modalities.

\section{Data Acquisition}

Data was acquired with a Minolta Vivid 910 range scanner. One $640 \times 4803 \mathrm{D}$ scan and one $640 \mathrm{x} 480$ color image are obtained near simultaneously. From 365 people that participated in two or more image acquisition sessions, there were 302 who had good 2D and 3D ear 
images in two or more sessions. No special instructions were given to the participants to make the ear images particularly suitable for this study, and 823 out of 2,342 images were dropped for various quality control reasons: 265 instances with hair obscuring the ear, 124 cases with artifacts due to motion during the scan, 91 with the person wearing earrings, and 343 cases with poor image quality in either the $3 \mathrm{D}$ and / or the $2 \mathrm{D}$. Using the Minolta scanner in the high resolution mode that researcher used may make the motion artifact problem more frequent, as it takes 8 seconds to complete a scan.

\section{E. Biometric System Modules}

Enrollment Unit: The enrollment module registers individuals into the ear biometric system database. During the phase, an ear biometric reader scans the individual's ear biometric characteristic to produce its digital representation.

Feature Extraction Unit: The module processes the input sample to generate a compact representation called the template, which is then stored in a central database or a smartcard issued to the individual.

Matching Unit: The module compares the current input with the template. If the system performs identity verification, it compares the new characteristics to the user's master template and produces a score or match value (one to one matching). A system performing identification matches the new characteristics against the master templates of many users resulting in multiple match values (one too many matching).

Decision Maker: The module accepts or rejects the user based on a security threshold and matching score.

\section{PREPROCESSING}

The purpose of the preprocessing is to minimize the variation in the acquired image, while keeping the characteristic features of the subject. Different preprocessing methods were applied to $2 \mathrm{D}$ intensity data and $3 \mathrm{D}$ range data [6].

\section{A. 2D Data Normalization}

Researcher performed the 2D data normalization in two steps. First is the geometric normalization. Ears were aligned using two manually identified landmark points. The distance between the two points was used for scale, which means that all the extracted ears have the same distance between the Triangular Fossa and the Incisure Intertragica Similarly, the orientation of the line between the two points is used for rotation. After normalization, the line between these two points is vertical in the xy plane. The second step is histogram equalization, which is used to compensate for lighting variation between images. These preprocessing steps are entirely analogous to those standard used in face recognition from 2D intensity images [4] and those used in previous PCAbased ear recognition using 2D intensity images.

\section{B. 3D Data Normalization}

The normalization discussed next applies to preparing the range image from the 3D data for the 3D PCA and 3D edge-based approaches. No preprocessing is applied for the $3 \mathrm{D}$ ICP.

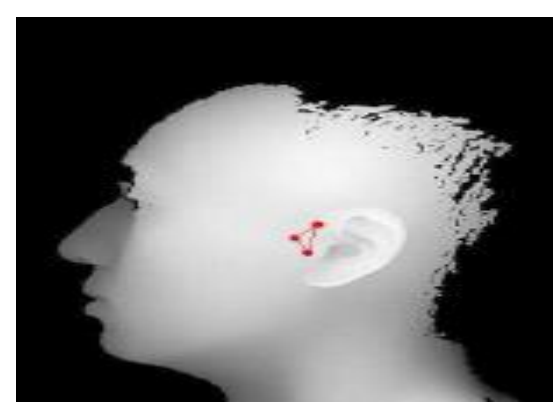

Figure 1. Three points used for plane fitting

3D image normalization is more complicated than 2D normalization, due to $\mathrm{z}$-direction rotation, holes and missing data [5]. Three steps of the 3D normalization are 3D pose normalization, pixel size normalization for the range images and whole filling. Normalization of 3D ear pose is required to create the range image for the $3 \mathrm{D}$ PCA and Hausdorff edge matching. In this study, the pose of the ear is determined by the orientation of the face plane connected with the ear. Three points are marked near the ear on the z-value image, as shown in Figure 1.

\section{Landmark Selection}

Researchers have investigated three different landmark selection methods. The first is the two-point landmark described in a study of "eigen-ears" with 2D intensity images. The upper point is the Triangular Fossa, and the lower point is the Antitragus, see Figure 2(a). However, researcher found that these two points are not easily detected in all images. For instance, many ears in the study have a small or subtle Antitragus. In order to solve this problem, two other landmark methods were conducted [7]. The second is similar to the first two-point landmark, but researcher used the Incisure Intertragica instead of Antitragus as the second point, as shown in Figure 2(b). The orientation of the line connecting these two points is used to determine the orientation of the ear, and distance between them is used to measure the size of the ear. The third method uses a two-line landmark, shown in Figure 2(c). One line is along the border between the ear and the face, and the other is from the top of the ear to the bottom. Unlike the two-point landmark, the two-line landmark promises to find the most part of the ear.

In the experiments, the second method is adopted for further ear extraction in PCA-based and edge-based algorithm, since it is good at blocking out background and avoiding ambiguity. The two-line landmark is used in the ICP-based algorithm, since it is better suited to the ICP algorithm properties. ICP uses the real 3D range data in the matching procedure and the two matching surfaces should overlap. The two-line landmark gives the 
opportunity to extract the whole ear for matching, but at the same time, it always includes some background, which increases the background variation, and affects the PCA-based and edge-based performance.
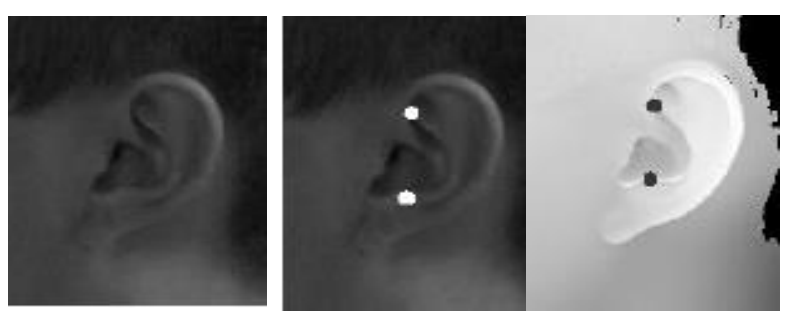

(a) Landmark 1: Using Triangular Fossa \& Antitragus
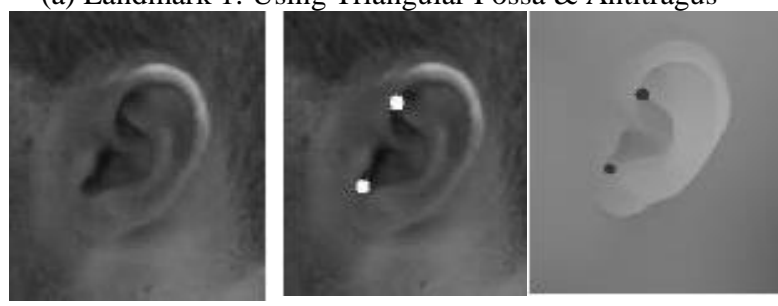

(b) Landmark 2: Using Triangular Fossa and Incisure Intertragica
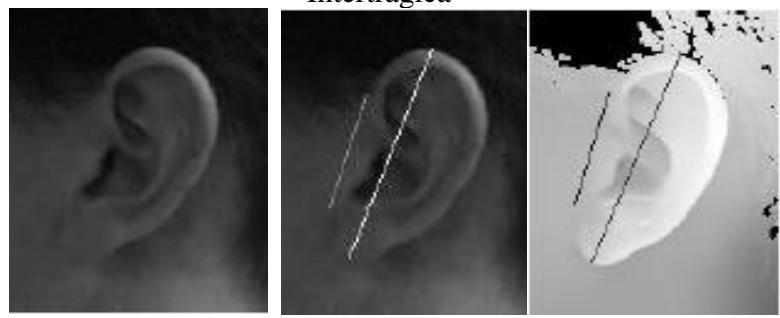

(c) Landmark 3: Using Two Lines

Figure 2. Example of ear landmarks

\section{Ear Extraction}

Ear extraction is based on the landmark locations on the original images. The original ear images are cropped to $(87 \times 124)$ for $2 \mathrm{D}$ and $(68 \times 87)$ for $3 \mathrm{D}$ ears. The reason for different ear size for the $2 \mathrm{D}$ and $3 \mathrm{D}$ data will be explained later.

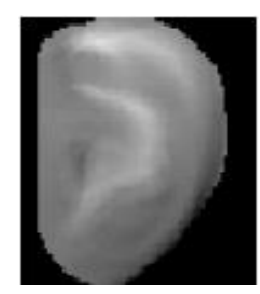

(a) Mask

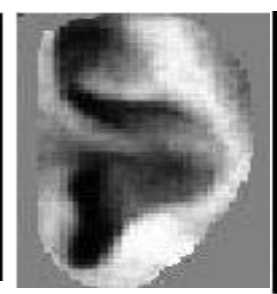

(b) 2D Intensity Ear

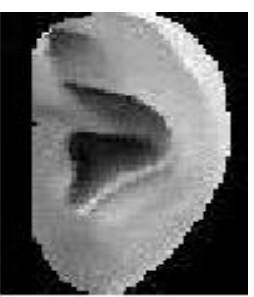

(c) 3D Depth Ear
Figure 3. Examples of ear mask and cropped 2D and 3D ear

The normalized images are masked to "gray out" the background and only the ear is kept. Figure 3 shows the mask and examples of the cropped 2D and 3D ear images.

\section{E. Hausdorff Range Edge Matching}

Achermann and Bunke [1] use an extension of the Hausdorff distance matching for the 3D face registration
[9]. Instead of using original 2D Hausdorff distance, they introduce a 3-D version of the partial Hausdorff distance. All the computation is based on the 3D space. In the experiment, the matching is between two edge images, therefore, only 2D Hausdorff distance is computed during the procedure. Researcher noticed that the 3D depth data looks much "cleaner" than the 2D intensity data.

\section{F. Voxel nearest Neighbors}

The most time consuming part of the ICP algorithm is finding the closest point. For each point on the probe surface, the algorithm needs to return the closest point on the gallery surface. By using these pairs of corresponding points, the ICP algorithm iteratively refines the transforms between two surfaces, and finds the translation and rotation to minimize the error distance.

\section{IV.IMPLEMENTATION OF SYSTEM}

Given a set of source points $\mathrm{P}$ and a set of model points $\mathrm{X}$, the goal of ICP is to find the rigid transformation $\mathrm{T}$ that best aligns $P$ with $X$. Beginning with a starting estimate $\mathrm{T} 0$, the algorithm iteratively calculates a sequence of transformations $\mathrm{Ti}$ until the registration converges. The algorithm computes correspondences by finding closest points, and then minimizes the mean square difference between the correspondences. A good initial estimate of the transformation is required, and all scene points are assumed to have correspondences in the model. The centroid of the extracted ear is used as a starting point in the experiments.

The general ICP algorithm requires no extracted features, or curvature computation [3]. The only preprocessing of the range data is to remove the outliers. In a 3D face image, the eyes and mouth are common places to cause holes and spikes. 3D ear images do exhibit some spikes and holes due to oily skin or sensor error, but much less than in the $3 \mathrm{D}$ face images. The initial experiment does not have outlier removal. Researcher also considers a version of ICP that does some outlier removal as part of the algorithm.

\section{A. Noise Removal}

Given a profile image, it is very difficult to isolate the ear without any background and noise around it. This problem will affect the ICP performance. One observation is that the noise mostly occurs on the top part of the ear. The bottom part of the ear is relatively clean, except when an earring appears. The blue line in the truth writing which goes through the ear top to the bottom, defines the bottom boundary of the ear clearly. Taking advantage of the fact that the ear edge is a continuous curve, researcher start from the bottom point, and use a seed-growing method to trace the ear edge and eliminate the noise.

\section{B. Speed Limitation}

It is well known that the basic ICP algorithm is effective but time consuming for 3D object registration. In order to make it more practical, it is necessary to speed 
up the algorithm. Two steps which are intended to make the algorithm faster are considered in this section. One is to control the number of iterations, and the other is to use appropriate data structures to shrink the running time. The number of iterations is initially set as 50, but researcher found the error distance decreases much faster in the first iteration than in the later iterations. So instead of using a fixed number of iterations, researcher measures the drop in the average distance between paired points between two consecutive iterations. Using a threshold of $0.0001 \mathrm{~mm}$, the average of the number of iterations decreases from 50 to 25.74 , and the performance stays the same.

\section{Outlier Elimination}

By using the ICP algorithm to align two surfaces, the quality of alignment highly depends on selecting good pairs of corresponding points from two surfaces. When outliers or missing points occur, their corresponding points will distract the alignment and generate the wrong position. For the ear biometric, hair is the most common causes of outliers, and some time the hair-cover is inevitable. Therefore outlier elimination becomes a requirement. An "outlier" match can occur when there is noise in one of the two point sets or when there is a poor match. To improve performance, outlier elimination is added to the original ICP implementation.

\section{2D Ear Data}

Two gallery/probe datasets with different scaling of the ear sizes are examined on 2D data. One is set as the actual size of the ear, and the other is set at 1.25 times the size of ear (see Figure 4). The performance of 2D regular ear size (Figure 4(a)), shown. The performance is lower than that reported by Chang in his study of 2D "eigenears". Looking closely at the images created from the eigenvectors associated with 3 largest Eigen values (Figure 5(a)), it was apparent that each of them had some space behind the contour of ear.

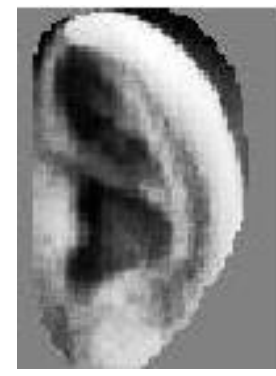

(a) Regular ear size

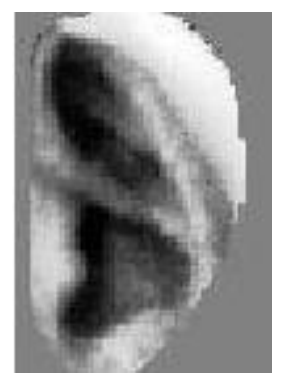

(b) 1.25 times of regular size
Figure 4. Experiments using different 2D ear size

This suggested enlarging the ear and so blocking out more background, which potentially causes the variation. After enlarging the ear to 1.25 times the original size (Figure 4(b)), there was no space behind the contour of ear in Figure 5(b). The rank-one recognition rate increased from $66.9 \%$ to $71.4 \%$ when using 202 subjects.

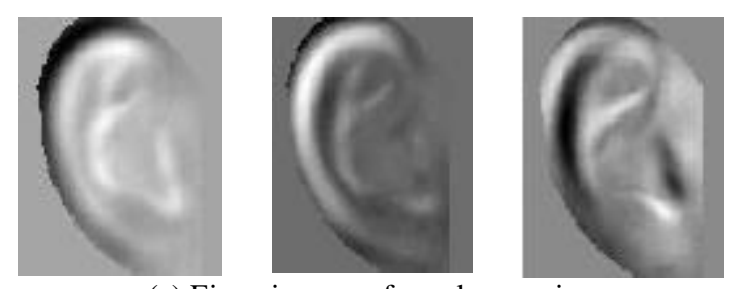

(a) Eigen images of regular ear size

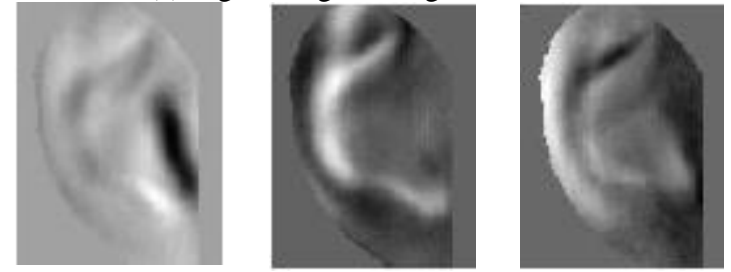

(b) Eigen images of enlarged ear size

Figure 5. Eigen ear images of Eigen vectors associated with 3 largest Eigen values

\section{E. 3D Ear Data}

Due to the $3 \mathrm{D}$ range data preprocessing, the size of a particular person's ear in pixels is constant over different images of that person. Therefore, no scale process is applied in the 3D ear extraction. Also, two different experiments were conducted on the $3 \mathrm{D}$ ear data. One is using the original ear range data. The other is applying mean and median filters on the original data to fill the holes of the cropped ear (see Figure 6). The rank-one recognition rate is improved from $58.4 \%$ to $64.8 \%$ with hole-filling when using 202 subjects. This is still not very good in an absolute sense. One possible reason is that the ear structure is quite complex, and so using mean and median filter alone might not be good enough to fill holes in the 3D range data. Applying whole filling on the 302 subjects, the performance stays at $55.3 \%$ rank one recognition rate.

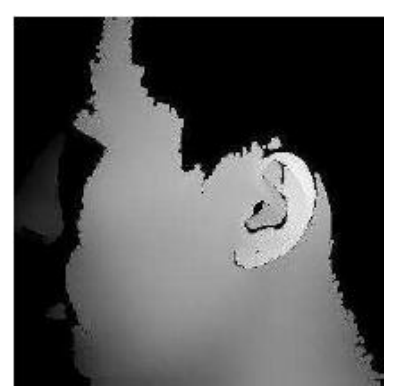

(a) Original range data with missing data.

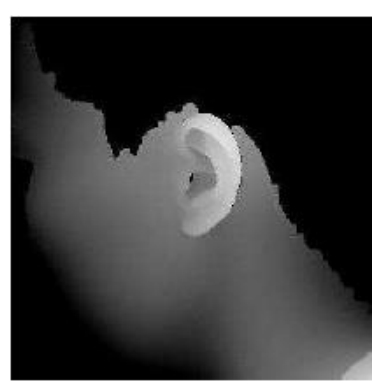

(b) After applying median and mean filters.
Figure 6: Hole filling for 3D range data

\section{F. Scaling with Dataset Size}

It has been suggested by that scaling of performance with dataset size is a critical issue in biometrics. When the gallery size becomes bigger, the possibility to get a false match increases. Usually, some techniques scale better to larger datasets than others. A good algorithm should keep the performance within a reasonable range when the data size expands. Here researcher focuses on 
comparing 2D PCA and 3D ICP. Table 4.5 shows the scalability of the 3D ICP and 2D PCA with different gallery sizes. When the gallery size is 25 , PCA has $92 \%$ rank one recognition, and ICP is at $100 \%$. As gallery size doubles, there is around a $10 \%$ drop in the PCA performance, and when the gallery has 302 subjects, the performance decreases to $63.8 \%$. However, ICP shows a much better scalability. When the gallery size doubles, there is less than $1 \%$ drop in ICP performance, and it still reaches $98.7 \%$ rank one recognition rate when the gallery size is 302 subjects. Checking all the incorrect matches for different gallery size, there is one image always mismatched. And of the new incorrect matches appeared in data size 302, two of them are new to all the other experiments using different data size, one of them drops from rank one to rank two when the data size increases from 200 to 302 [8].

\section{TESTING BIOMETRIC SYSTEM}

A summary of the more common of these tests is described below:

1) Acceptance Testing: The process of determining whether an implementation satisfies acceptance criteria and enables the user to determine whether or not to accept the implementation. This includes the planning and execution of several kinds of tests (E.Q., functionality, quality, and speed performance testing) that demonstrate that the implementation satisfies the user requirements.

2) Conformity: Fulfillment by a product, process or service of specified requirements.

3) Interoperability Testing: The testing of one implementation (product, system) with another to establish that they can work together properly.

4) Performance Testing: Measures the performance characteristics of an Implementation under Test (IUT) such as its throughput, responsiveness, etc., under various conditions.

5) Robustness Testing: The process of determining how well an implementation processes data which contains errors.

\section{A. Biometric Performance Measurements}

The performance of biometric system is tested usually in terms of False Rejection Rate (FRR), False Acceptance Rate (FAR), and Failure to Enroll Rate (FER), Enrollment Time, and Verification Time. The false acceptance rate is most important when security is a priority whereas low false rejection rates are favored when convenience is the priority [3].

The biometric system employed in the flight deck must have a low false acceptance rate since security is the priority. If the false acceptance rate is a low as possible then researcher have better chance of not allowing unauthorized subjects into the system. The point at which the FAR and FRR meet or crossover is known as the equal error rate. This rate gives a more realistic measure of the performance of the biometric system rather than using either the FAR or FRR individually.

\section{VI.EXPERIMENTAL RESULTS}

The ideas described in the preceding sections have been implemented in $\mathrm{C}++$, and incorporated into the ICP matching program. In order to evaluate the efficiency of this method, researcher compares the performance, space and running time between the original algorithm and the new incorporated ICP matching. Both experiments use ear range data from 302 subjects. For each of the 302 subjects, the earlier 3D images are used for galleries, and the later 3D images are used as probes. All the gallery images use the full resolution, and the probes are subsampled by every 4 rows and every 4 columns. In addition, different voxel sizes are tested, and results are presented. The system runs on quad processor Pentium Xeon $2.8 \mathrm{GHz}$ machines with 2GB RAM. However, for very large galleries the voxel approach yields an enormous improvement in speed. In a real biometrics application, some or the entire gallery might be kept in memory all the time.

\section{A. Ear Symmetry Experiment}

One less controlled approach is matching a mirrored left or right ear, which means that for one subject we enroll his right ear and try to recognize his left ear. Two different angles of view have been examined. They are 30 degree off the center and 45 degree off the center. In the initial data processing includes landmarks ground truth and ear extraction.

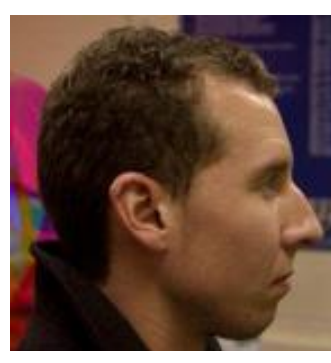

(a) Right Ear

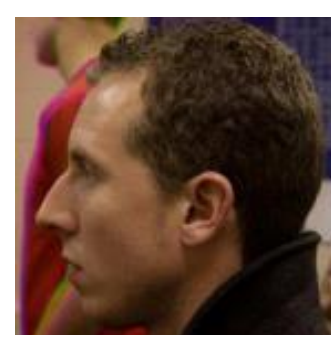

(b) Left Ear
Figure 7. Image acquired for Ear Symmetric experiments.

The initial results are presented in Table 1. The right ear of the subject is used as the gallery, and the left ear is used as the probe, see Figure 7. Both ear images are taken on the same day. By analyzing the results, we found that most people's left and right ears are symmetric or at least very similar. But some people's lefts and right ears have different shapes.

TABLE 1. PERFORMANCE OF EAR SYMMETRY EXPERIMENTS

\begin{tabular}{|c|c|c|}
\hline & Number of Subjects & Performance \\
\hline 30 degree off & 88 & $90 \%$ \\
\hline 45 degree off & 119 & $90 \%$ \\
\hline
\end{tabular}




\section{B. Off-Angle Experiment}

Another dim. ension of variability is the angle of view, which means that the enrolled gallery ear and the probe ear are from different angles of view. We enroll a straight-on right ear, and try to recognize a right ear viewed at some different amount of angle. The initial experiments only test on a small dataset. We have some observations, but cannot draw any strong conclusions until we have a larger dataset.

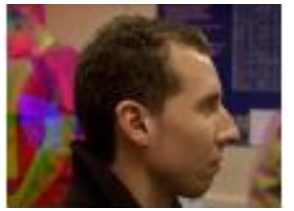

(a) Straight-on

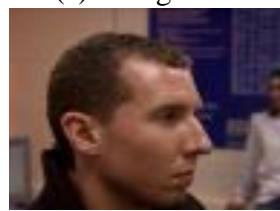

(c) 30 Degree off

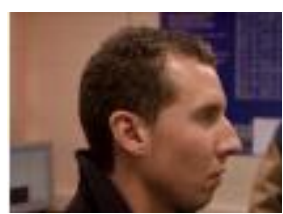

(b) 15 Degree off

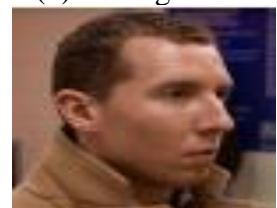

(d) 45 Degree off
Figure 8: Image acquired for Off Angle Experiments

In this experiment, there are 4 different angles of view for each ear: straighton, 15 degree off center, 30 degree off center and 45 degree off center, as shown in figure 8 . The 45 degree images were taken on the first week. The 30 degree images were taken the second week. Finally, the 15 degree and straight-on images were both taken on the third week. For each angle of ear image, we match it against all images in the different angle datasets.

TABLE 2. OFF ANGLE EXPERIMENTS WITH A 24SUBJECT DATASET

\begin{tabular}{|l|l|l|l|l|}
\hline Straight-on & $15^{\circ}$ off & $30^{\circ}$ off & $45^{\circ}$ off & Average \\
\hline $15^{\circ}$ off & $100 \%$ & $87.5 \%$ & $70.8 \%$ & $86.1 \%$ \\
\hline $30^{\circ}$ off & $100 \%$ & $100 \%$ & $87.5 \%$ & $95.8 \%$ \\
\hline $45^{\circ}$ off & $87.5 \%$ & $100 \%$ & $95.8 \%$ & $94.4 \%$ \\
\hline Average & $88.9 \%$ & $95.8 \%$ & $95.8 \%$ & $84.7 \%$ \\
\hline
\end{tabular}

There were only 24 subjects that showed up on every week. Therefore the initial experimental results, shown in Table 2, are obtained from a fairly small dataset. Two observations are drawn from the Table 2. The first is that 15 degree and 30 degree off center have better overall performance than the straight on and 45 degree off center. This observation makes sense, since there is more ear area exposed to the camera when the face is 15 degrees and 30 degrees off center. Also matching is generally good for 15 degrees off or less, but gets worse for more than 15 degrees. The second observation is that the elements in table 2 are symmetric. A larger dataset is required if we want to verify our observation and draw any conclusions. In a real biometrics application, some or the entire gallery might be kept in memory all the time.
This technique is aimed at a particular application in human identification. The idea is based on the possibility of computing before the real matching procedure taking place. Different voxel sizes are examined, and the performance and running time are compared with the results from the original ICP algorithm. Our experimental results verify the expected feature of our approach. The online matching time drops significantly when we use the pre-computed results from the offline computation. Our results demonstrate that for very large galleries the voxel approach yields an dramatic improvement in speed. While we only address the problem using 3D ear data, it would be interesting to investigate whether the proposed fast ICP-based method is efficient in other applications, like $3 \mathrm{D}$ face or finger recognition.

\section{CONCLUSIONS}

The main contribution of this chapter is the "Precomputed Voxel Closest Neighbors" strategy to improve the speed of the original ICP algorithm. This technique is aimed at a particular application in human identification. The idea is based on the possibility of computing before the real matching procedure taking place. Different voxel sizes are examined, and the performance and running time are compared with the results from the original ICP algorithm. The experimental results verify the expected feature of the approach. The online matching time drops significantly when researcher use the pre-computed results from the offline computation. The results demonstrate that for very large galleries the voxel approach yields a dramatic improvement in speed. While researcher only address the problem using 3D ear data, it would be interesting to investigate whether the proposed fast ICP-based method is efficient in other applications. By implementing reasonable safeguards, researcher can harness the power of the technology to maximize its public safety benefits while minimizing the intrusion on individual privacy.

\section{REFERENCES}

[1] B. Bhanu and H. Chen, Human ear recognition in 3D In Workshop on Multimodal User Authentication, pages 91-98 (2003).

[2] M. Burge and W. Burger, Ear biometrics. In Biometrics: Personal Identification in Networked Society, pages 273-286, Kluwer Academic (1999).

[3] M. Burge and W. Burger, Ear biometrics in computer vision. In 15th International Conference of Pattern Recognition, volume 2, pages 822-826 (2000).

[4] K. Chang, K. Bowyer and V. Barnabas, Comparison and combination of ear and face images in appearance-based biometrics. In IEEE Trans. Pattern Anal. Machine Intell. Volume 25, pages 1160-1165 (2003).

[5] K. Chang, K. Bowyer and P. Flynn, Face recognition using 2D and 3D facial data. In Workshop on 
Multimodal User Authentication, pages 25-32 (2003).

[6] A. Iannarelli, Ear identification. In Forensic identification series, Fremont, California, Paramont Publishing Company (1989).

[7] B. Victor, K. Bowyer and S. Sarkar, An evaluation of face and ear biometrics. In 16th International Conference of Pattern Recognition, pages 429-432 (Aug. 2002).

[8] K. Pulli, Multiview registration for large data sets. In Second International Conference on 3-D Imaging and Modeling (3DIM '99), pages 160-168 (October 04-08, 1999).

[9] D. Huttenlocher, G. Klanderman and W. Rucklidge, Comparing images using the hausdorff distance. In IEEE Trans. Pattern Anal. Machine Intell. Volume 15(9), pages 850-863 (1993).

First Author Profile:

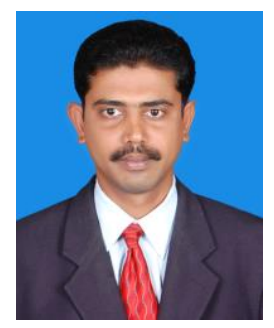

Mr. V.K. NARENDIRA KUMAR M.C.A., M.Phil., Assistant Professor, Department of Information Technology, Gobi Arts \& Science College (Autonomous), Gobichettipalayam - 638 453, Erode District, Tamil Nadu, India. He received his M.Phil Degree in Computer Science from Bharathiar University in 2007.
He has author more than 40 international journal article publications. He has authored or co-authored more than 60 technical papers and conference presentations. He is an editorial board member for several scientific international journals. His research interests are focused on Internet Security, Biometrics, Advanced Networking, Visual Human-Computer Interaction, and Multiple Biometrics Technologies.

Second Author Profile:

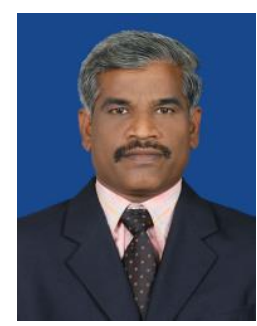

Dr. B. SRINIVASAN M.C.A., M.Phil., M.B.A., Ph.D., Associate Professor, PG \& Research Department of Computer Science, Gobi Arts \& Science College (Autonomous), Gobichettipalayam 638 453, Erode District, Tamil Nadu, India. He received his Ph.D. Degree in Computer Science from Vinayaka Missions University in 11.11.2010. He has author or coauthored more than 45 international journal article publications. He has authored or co-authored more than 75 technical papers and conference presentations. He is a reviewer for several scientific e-journals. His research interests include automated biometrics, computer networking, Internet security, and performance evaluation.

How to cite this paper: V.K. Narendira Kumar, B. Srinivasan,"Automated Human Identification Scheme using Ear Biometrics Technology", IJIGSP, vol.6, no.3, pp. 58-65, 2014.DOI: 10.5815/ijigsp.2014.03.08 